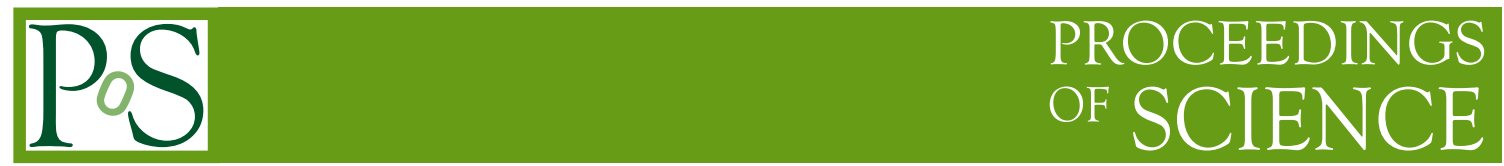

\title{
Results from combined CMS-TOTEM data
}

\author{
Sercan Sen ${ }^{* \dagger}$ \\ Department of Physics Engineering, Hacettepe University, Ankara, Turkey \\ The University of Iowa, Iowa City, USA \\ E-mail: Sercan. Sen@cern.ch
}

\begin{abstract}
The combined data taking of the CMS and TOTEM experiments allows to characterize hadronic final states in an extremely wide pseudo-rapidity range and opens up a window to a rich diffractive/forward physics program. In this paper, the trigger strategy of the common data taking is described, and the physics results from combined CMS-TOTEM data is presented.
\end{abstract}

XXIV International Workshop on Deep-Inelastic Scattering and Related Subjects

11-15 April, 2016

DESY Hamburg, Germany

\footnotetext{
* Speaker.

${ }^{\dagger}$ On behalf of the CMS and TOTEM Collaborations.
} 


\section{Introduction}

The CMS and TOTEM experiments share the same interaction point (IP) at the LHC and that makes it possible to carry out a joint diffractive and forward physics programme. This programme includes soft diffraction, as well as hard diffraction and exclusive studies in various physics channels with unprecedented measurement possibilities. The programme is already well established for the low, medium, and high luminosity running conditions of LHC [1, 2].

The CMS apparatus features a superconducting solenoid providing a uniform magnetic field of 3.8 T. Within the field volume are a silicon tracker system, an electromagnetic and hadronic calorimeter. The calorimeter system surrounds the tracker and covers the pseudorapidity region up to $|\eta|=3$. The forward hadron calorimeters (HF) extend the calorimetry coverage up to $|\eta|=5.2$. The very forward calorimeter, CASTOR, is placed only at one side of CMS in the region $-6.6<$ $\eta<-5.2$. Forward Shower Counter (FSC) detectors are located on both sides of the IP, in the region $6 \lesssim|\eta| \lesssim 8$, and they are designed to detect showers produced by particles hitting the beam pipes and nearby material. The muon system allows reconstruction and identification of muons in the $|\eta|<2.4$ region. Events are recorded using a two-level trigger system; Level-1 (L1) and highlevel trigger (HLT) systems. A complete description of the CMS detector and the trigger system can be found in [3].

The TOTEM experiment [4] comprises three subdetectors: the T1, T2 forward trackers and a series of Roman Pot (RP) stations. The T1 and T2 are placed symmetrically on each side of the IP in the pseudorapidity regions $3.1 \leq|\eta| \leq 4.7$ and $5.3 \leq|\eta| \leq 6.5$, respectively. The RPs, special movable beam-pipe instruments which can detect leading protons scattered at very small angles, are placed at $\pm 147 \mathrm{~m}$ and $\pm 220 \mathrm{~m}$ from the IP.

\section{CMS-TOTEM joint data taking at high $\beta^{*}$}

The first CMS-TOTEM joint data taking with common triggers was performed in 2012, during a dedicated run with low pile-up and a non-standard $\beta^{*}=90 \mathrm{~m}$ optics configuration, where $\beta^{*}$ is the amplitude function of the beam at the IP. The data were taken by each experiment with a bidirectional exchange of triggers; TOTEM RPs \& T2 to CMS, and CMS jet \& lepton triggers to TOTEM. The same events were saved by both experiments. The events reconstructed at CMS and at TOTEM are then synchronized offline by requiring the same LHC orbit and bunch numbers. The data correspond to an integrated luminosity of $45 \mu b^{-1}$, and are mainly useful to perform minimum bias measurements such as forward particle production. The data also showed some sensitivity to central exclusive processes but higher statistics were needed for a precise measurement. A brief overview of the analyses performed with the 2012 data is given in the following section.

In 2015, the second running period of LHC (Run II) started at a new record collision energy, $13 \mathrm{TeV}$. The CMS-TOTEM trigger strategy was similar to the one in 2012: the CMS (TOTEM) contributed to TOTEM (CMS) trigger decision, and initiated simultaneous readout of both the CMS and TOTEM detectors. A sketch of several types of CMS-TOTEM triggers which were implemented for 2015 data taking $\left(\beta^{*}=90 \mathrm{~m}\right)$ is given in Fig. 1 .

These dedicated triggers and the significantly increased luminosity $\left(\sim 0.4 \mathrm{pb}^{-1}\right)$ in 2015 , allow to study various diffractive and exclusive processes such as exclusive low mass resonance 


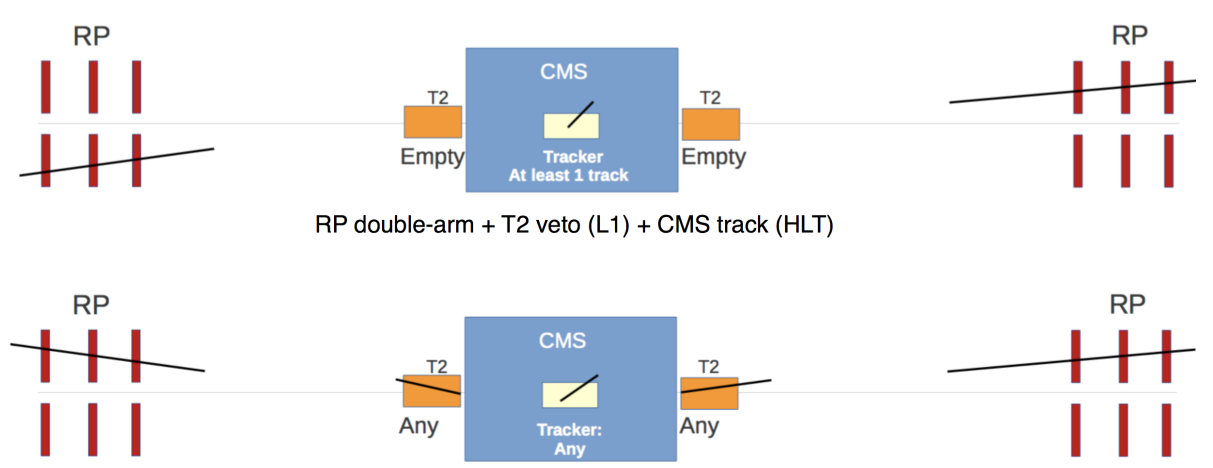

RP double-arm Top-Top OR Bottom-Bottom, T2 any (L1), CMS track any (HLT)

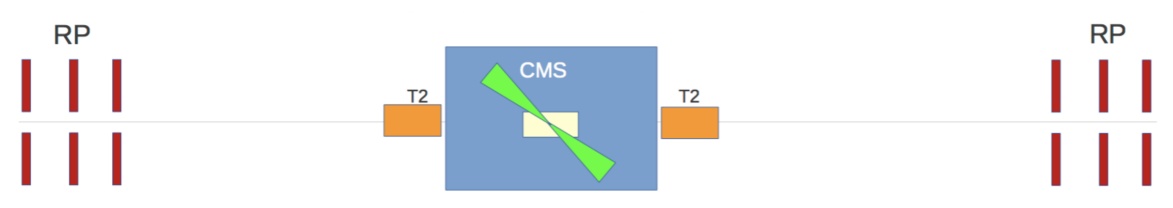

CMS only (L1): dijet with $p_{\mathrm{T}}^{\text {jets }}>20 \mathrm{GeV}$ or dimuon

Figure 1: A sketch of CMS-TOTEM triggers implemented for 2015 data taking at high $\beta^{*}$.

production, high mass central diffraction, and central exclusive jet production. The overall statistics for $\beta^{*}=90 \mathrm{~m}$ runs in 2015 is an order of magnitude larger with respect to 2012 data. The CMS-TOTEM common software tools are updated and validated successfully for the reprocessing of the new data. A huge part of the datasets is already merged and the physics analyses are started. The potential of central exclusive production studies in high $\beta^{*}$ runs with CMS-TOTEM data in pp collisions at $13 \mathrm{TeV}$ is discussed in detail in [5]. In addition, the CMS-TOTEM feasibility studies for single diffractive $Z, W, J / \psi$ and central exclusive dijet production can be found in [6].

\section{Physics highlights of Run I}

\subsection{Charged particles distributions}

The pseudorapidity distributions of charged particles in pp collisions at $\sqrt{s}=8 \mathrm{TeV}$ are measured in the ranges $|\eta|<2.2$ and $5.3<|\eta|<6.4$ by the CMS and TOTEM experiments [7]. The data were collected during a CMS-TOTEM data taking in 2012. A minimum bias trigger was provided by TOTEM (presence of T2 track on either side). In Fig. 2, the combined CMS-TOTEM charged-particles pseudorapidity distributions at $\sqrt{s}=8 \mathrm{TeV}$ are given for three event classes: a sample of inclusive inelastic pp events, a sample dominated by non single diffractive dissociation (NSD) events, and a sample enriched in single diffractive dissociation (SD) events. The error bars correspond to the statistical uncertainty and the uncorrelated systematics between neighbouring bins. The uncertainty band represents the combined systematics and statistical uncertainties.

The results indicate that the forward pseudorapidity density decreases with $|\eta|$. The predictions from various MC event generators differ from the data by up to about $\pm 30 \%$. None of the models are able to describe the data in the full- $|\eta|$ range and for all event topologies. The QGSJETII04 model describes the inclusive and NSD-enhanced samples both in central and forward region, but it underestimates the SD-enhanced data. 

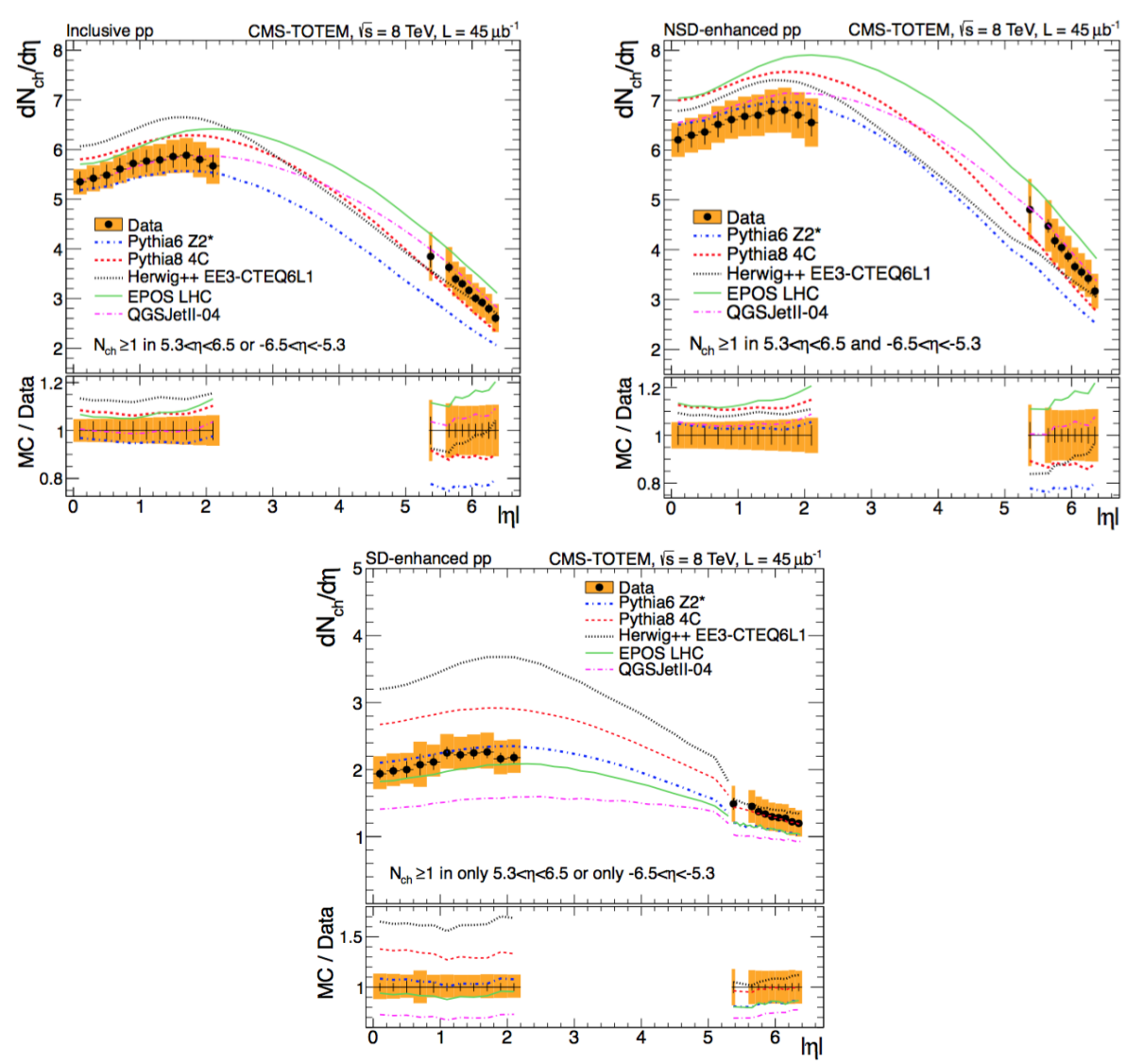

Figure 2: The combined CMS-TOTEM charged-particles pseudorapidity distributions at $\sqrt{s}=8 \mathrm{TeV}$ for inclusive (top left), NSD-enhanced (top right), and SD-enhanced (bottom) samples [7].

The midrapidity particle density is expected to follow a power-law dependence, $\mathrm{d} N_{\mathrm{ch}} /\left.\mathrm{d} \eta\right|_{\eta=0} \propto s^{\varepsilon}$, with $\varepsilon$ in the range $0.14-0.24$. In Fig 3, the average value of $\mathrm{d} N_{\mathrm{ch}} / \mathrm{d} \eta$ at $\eta \approx 0$ as a function of centerof-mass energy is shown, together with the data from various other experiments for NSD events in $p p$ and $p \bar{p}$ collisions. The dashed line in the figure represents the power-law fit to the data. A value of $\varepsilon=0.23 \pm 0.01$ is obtained after fitting.

The measurement of the charged particles distributions over $|\eta|<6.4$ is a very important example that puts strong constraints on interaction models, and provides great inputs for tuning model parameters of MC event generators [8].

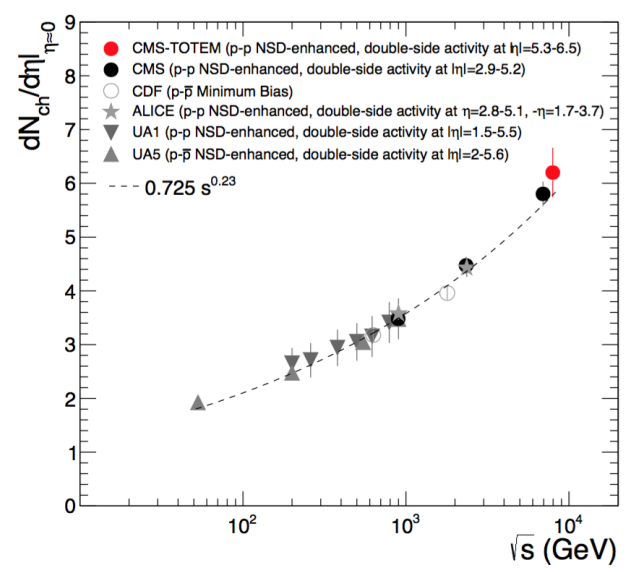

Figure 3: Average value of $\mathrm{d} N_{\mathrm{ch}} / \mathrm{d} \eta$ at $\eta \approx 0$ as a function of $\sqrt{s}$ in $\mathrm{pp}$ and $\mathrm{p} \overline{\mathrm{p}}$ collisions [7]. 


\subsection{High- $p_{\mathrm{T}}$ jets with two leading protons}

Central high- $p_{\mathrm{T}}$ jet production with two leading protons, $\mathrm{pp} \rightarrow \mathrm{p}+\mathrm{jets}+\mathrm{p}$, is also studied using CMS-TOTEM data at $\sqrt{s}=8 \mathrm{TeV}[9,10]$. Event selection requires two non-elastic protons detected in RPs on both sides of the CMS detector, in addition to jets reconstructed with $p_{\mathrm{T}}>20$ $\mathrm{GeV}$ in $|\eta|<4.4$ at CMS. Also the CMS FSC detectors are required to be empty to reject the QCD background. An event display of a three jets event with two leading protons is shown in Fig. 4.
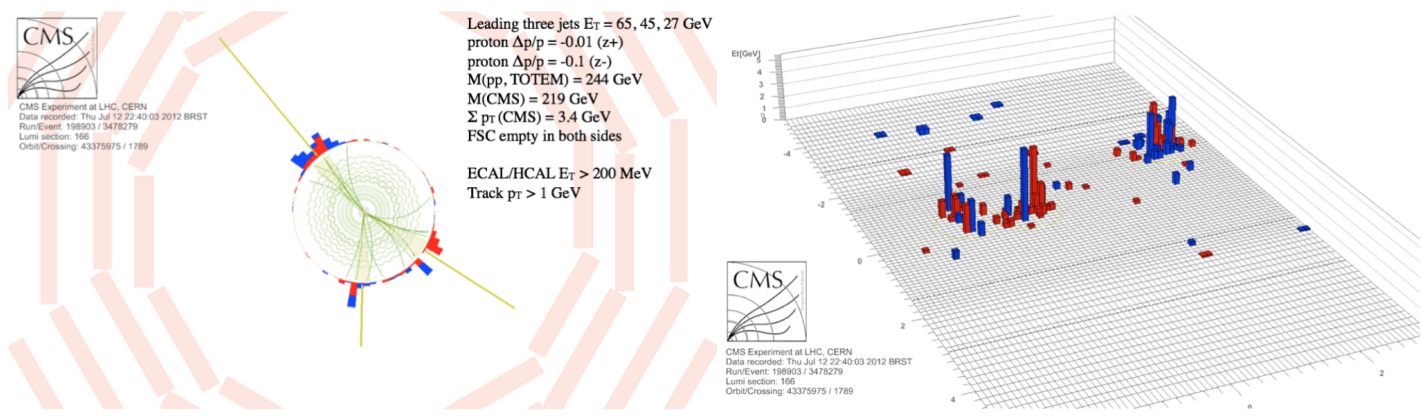

Figure 4: A central high- $p_{\mathrm{T}}$ jets event with two leading protons detected in RPs [9].

\subsection{A data driven method to suppress pile-up and beam-halo backgrounds}

The non-diffractive events, pile-up events and beam-halo are important sources of background for diffractive analyses. The background from non-diffractive events can largely be suppressed when leading protons are detected in RPs. However, protons originating from pile-up or particles from beamhalo hitting to the RPs can cause misidentification of diffractive events. In order to suppress this background, a data driven method is developed using CMS-TOTEM Run I data [11]. In the method, the difference between the longitudinal momentum loss of the proton reconstructed with CMS ( $\xi_{\mathrm{CMS}}$, calculated by summing up the energies and

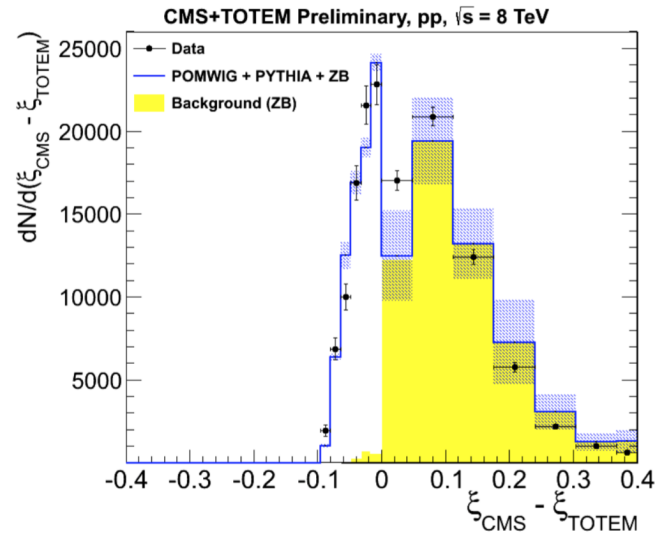

Figure 5: Distribution of the difference between $\xi_{\mathrm{CMS}}$ and $\xi_{\text {TOTEM }}$ in single diffractive dijet production $(\mathrm{pp} \rightarrow \mathrm{pjjX})$ [11]. longitudinal momenta of the final state particles in CMS) and that reconstructed with TOTEM RPs ( $\left.\xi_{\text {TOTEM }}\right)$ is used to suppress the pileup and beam-halo backgrounds. The distribution of the difference between $\xi_{\mathrm{CMS}}$ and $\xi_{\text {TOTEM }}$ in single diffractive dijet production (CMS-TOTEM Run I data), is shown in Fig. 5. The background events are populated in $\xi_{\mathrm{CMS}}-\xi_{\text {TOTEM }}>0$ region which is kinematically forbidden. The $\xi_{\mathrm{CMS}}-\xi_{\text {TOTEM }}<0$ requirement thus selects mostly the signal events. The remaining background contribution was found to be $\sim 4 \%$. The method is also applicable to 2015 data since the running conditions are similar to the ones in 2012. 


\section{Acknowledgements}

This work was supported by Hacettepe University, Scientific Research Projects Program through the Project: FBB-2016-10069.

\section{References}

[1] M. Albrow et al. [CMS and TOTEM diffractive and forward physics working Group Collaboration], "Prospects for Diffractive and Forward Physics at the LHC," CERN-CMS-Note-2007-002, CERN-LHCC-2006-039-G-124, CMS-Note-2007-002, TOTEM-Note-2006-005, LHCC-G-124, CERN-TOTEM-Note-2006-005.

[2] K. Akiba et al. [LHC Forward Physics Working Group Collaboration], "LHC Forward Physics," CERN-PH-LPCC-2015-001, SLAC-PUB-16364, DESY-15-167 (2015).

[3] S. Chatrchyan et al. [CMS Collaboration], "The CMS experiment at the CERN LHC," JINST 3, S08004 (2008). doi:10.1088/1748-0221/3/08/S08004

[4] G. Anelli et al. [TOTEM Collaboration], "The TOTEM experiment at the CERN Large Hadron Collider,” JINST 3, S08007 (2008). doi:10.1088/1748-0221/3/08/S08007

[5] K. Österberg [TOTEM Collaboration], "Potential of central exclusive production studies in high $\beta^{*}$ runs at the LHC with CMS-TOTEM,” Int. J. Mod. Phys. A 29, no. 28, 1446019 (2014). doi:10.1142/S0217751X14460191

[6] CMS Collaboration, TOTEM Collaboration, "CMS-TOTEM feasibility studies for single diffractive $Z, W, J / \psi$ and central exclusive dijet production in pp collisions at $\sqrt{s}=13 \mathrm{TeV}$ ", CERN-TOTEM-NOTE-2014-002, CMS-PAS-FSQ-14-001.

[7] S. Chatrchyan et al. [CMS and TOTEM Collaborations], "Measurement of pseudorapidity distributions of charged particles in proton-proton collisions at $\sqrt{s}=8 \mathrm{TeV}$ by the CMS and TOTEM experiments," Eur. Phys. J. C 74, no. 10, 3053 (2014) doi:10.1140/epjc/s10052-014-3053-6 [arXiv:1405.0722 [hep-ex]].

[8] V. Khachatryan et al. [CMS Collaboration], "Event generator tunes obtained from underlying event and multiparton scattering measurements," Eur. Phys. J. C 76, no. 3, 155 (2016) doi:10.1140/epjc/s10052-016-3988-x [arXiv:1512.00815 [hep-ex]].

[9] CMS and TOTEM Collaborations, “CMS-TOTEM event display: high- $p_{T}$ jets with two leading protons", CMS-DP-2013-004.

[10] CMS and TOTEM Collaborations, "Central high- $p_{T}$ jet production during low pile-up, high $\beta^{*}$ run at $\sqrt{s}=8$ TeV”, CMS-DP-2013-006.

[11] CMS and TOTEM Collaborations, "Development of a beam-halo and pileup background rejection procedure for diffractive analyses in low-pileup runs based on Roman Pot tagging with the CMS and TOTEM experiments", CMS-DP-2015-005. 